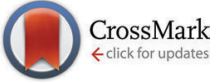

Cite this: Phys. Chem. Chem. Phys., 2016, 18, 20802

Received 31st May 2016 Accepted 24th June 2016

DOI: $10.1039 / c 6 c p 03742 a$

www.rsc.org/pccp

\title{
Photoionization access to cyclodextrin-encapsulated resveratrol phenoxy radicals and their repair by ascorbate across the phase boundary
}

\author{
Christoph Kerzig and Martin Goez*
}

\begin{abstract}
Repair reactions of phenoxy radicals by co-antioxidants are key parts of radical scavenging cascades in nature. Yet, kinetic and mechanistic studies of such repairs are scarce, particularly at biologically relevant interfaces. For the popular red-wine polyphenol resveratrol, we present the first example of repairing a cyclodextrin-complexed phenoxy radical by a water soluble co-antioxidant (ascorbate), a reaction of practical importance given the fact that both antioxidants and cyclodextrins are large-scale food additives. To prepare the phenoxy radical from its parent compound inside the cavities of native or hydroxypropylsubstituted $\alpha$ - and $\beta$-cyclodextrins, we employed laser photoionization with UV-A (355 nm), which does not rely on additional reagents, and therefore leaves the repair completely undisturbed. A global fit of the intensity dependence pinpoints the cyclodextrin influences on the biphotonic resveratrol ionization as a shift of the ground-state absorption spectrum and a longer life of the first excited state due to the suppression of the geometrical isomerization by the rigid containers, whereas the actual electron ejection from an upper excited state is almost medium-independent. The exchange of the phenoxy radical between the cyclodextrin interior and the aqueous bulk is immeasurably slow on the timescale of its repair by the ascorbate monoanion. Kinetic H/D isotope effects and activation entropies identify the repair at the cyclodextrin-water interface as a concerted proton-electron transfer with no mechanistic difference to homogeneous aqueous solution. The activation enthalpies reveal a steric repulsion between ascorbate and cyclodextrin that indicates a deeper embedding of the less hydrophilic phenoxy radical in the macrocycle compared to the parent compound, with the observed structure-rate relationships explainable on the basis of the cavity diameter and depth.
\end{abstract}

\section{Introduction}

Resveratrol (ResOH; for the structure, see Fig. 1b) is a red-wine polyphenol whose antioxidant properties are thought to be responsible for an astonishing variety of health benefits, ${ }^{1}$ kindling continuously growing interest in its uses both for medical treatment ${ }^{2}$ and as a dietary supplement. ${ }^{3}$ To overcome the poor water solubility of this lipophilic "wonder molecule", which inhibits its oral uptake, cyclodextrins are receiving increasing attention as its carriers. ${ }^{4-12}$ An alternative way of increasing the bioavailability of $\mathbf{R e s O H}$ is the addition of co-antioxidants, ${ }^{13}$ which themselves might not be as effective as ResOH in scavenging free radicals but can reconvert the resulting phenoxy radical $\mathbf{R e s O}^{\bullet}$ to $\mathbf{R e s} \mathbf{O H}$, the most important hydrophilic co-antioxidant for this purpose being ascorbate HAsc ${ }^{-}$ (vitamin C). Elucidating the kinetic and mechanistic aspects of these so-called repair reactions is essential for understanding radicalscavenging cascades as they occur in nature. ${ }^{14}$

Martin-Luther-Universität Halle-Wittenberg, Institut für Chemie, Kurt-Mothes-Str. 2, D-06120 Halle (Saale), Germany. E-mail: martin.goez@chemie.uni-halle.de
Although several reports have addressed radical scavenging by cyclodextrin-complexed ResOH,,$^{7,9,15-17}$ nothing whatsoever is known about the repair of $\mathbf{R e s O}^{\bullet}$ at the water-cyclodextrin interface. Even for $\alpha$-tocopherol (vitamin E), the best-investigated lipophilic antioxidant, kinetic and mechanistic studies on the repair of its phenoxy radical have focused exclusively on homogeneous solutions ${ }^{14,18-23}$ and model membranes. ${ }^{19-24}$ This lack of information concerning reactions across the water-cyclodextrin phase boundary-despite the widespread use of both cyclodextrins and antioxidants in food processing-prompted us to examine them using HAsc $^{-}$as the repairing agent for ResO'.

To this end, we have employed our recently developed ${ }^{25}$ conversion of ResOH into $\mathbf{R e s O}^{\bullet}$ without the participation of any auxiliary reagent through laser photoionization with UV-A $(355 \mathrm{~nm})$ to generate the radical inside the cavities of native and modified cyclodextrins (for the structures, see Fig. 1a) and to study its subsequent reaction with $\mathbf{H A s c}^{-}$, which can only reside in the aqueous phase. As we will show, the cyclodextrin containers influence both the photoionization and the repair reaction decisively through the geometric constraints they 
(a)

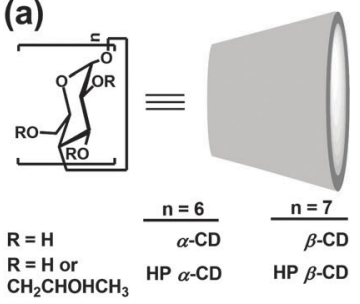

(b)

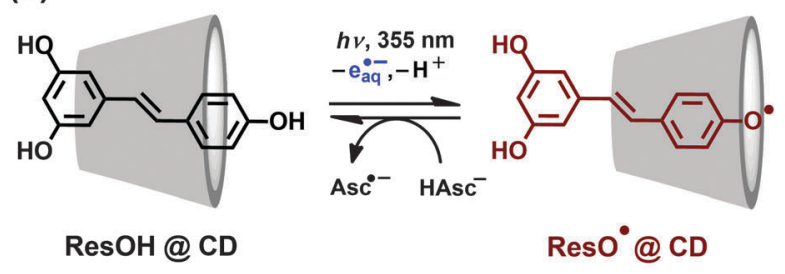

Fig. 1 (a) Schematic structures and abbreviations of the cyclodextrins used in this study. In the hydroxypropyl (HP) cyclodextrins, each glucose unit contains about one HP group. (b) Photoionization of cyclodextrin-complexed resveratrol (ResOH) and repair reaction of its phenoxy radical (ResO*) by the water-soluble ascorbate monoanion $\left(\mathrm{HAsc}^{-}\right.$) across the cyclodextrin-water interface.

impose, but they affect neither the quantum yield of the actual ionization step nor the repair mechanism, and their different effects on radical scavenging by $\mathbf{R e s O H}$ and on the repair of ResO $^{\bullet}$ by HAsc $^{-}$can be traced back to a deeper embedding of the more hydrophobic phenoxy radical in their interior.

\section{Results and discussion}

\subsection{Complexation of resveratrol with cyclodextrins and properties of the inclusion complexes}

The promising increase of both solubility and stability of ResOH upon its complexation by cyclodextrins has stimulated studies of its inclusion in these cavities by a variety of methods (phase solubility ${ }^{4,6,7,9}$ fluorescence, ${ }^{6,8,11}$ enzymatic oxidation, ${ }^{6}$ HPLC, ${ }^{5}$ isothermal titration calorimetry, ${ }^{10} \mathrm{NMR}^{12}{ }^{12}$ and circular dichroism ${ }^{12}$ ). However, these investigations often gave substantially divergent results even when similar experimental procedures were used: e.g., values between $1600 \mathrm{M}^{-14}$ and $18000 \mathrm{M}^{-16}$ were reported for the complexation constant of ResOH by HP $\beta$-CD. These discrepancies prompted us to (re)determine the binding constants of ResOH to all cyclodextrins employed in this work spectrophotometrically. This well-established method ${ }^{26}$ does not seem to have been applied to these systems although in the case of ResOH it is much more sensitive than fluorescence. From measurements of the fluorescence quantum yields, in conjunction with the absorption and luminescence spectra, we further obtained the excited-state lifetimes, which reflect how the encapsulation affects the photoinduced cis-trans isomerization.

2.1.1 Absorption. The absorption change of ResOH upon complexation provides a much more sensitive observable than does the fluorescence, which has a very low quantum yield (for comparison, see Section 2.1.2) leading to a poor signal-to-noise ratio. The addition of cyclodextrins shifts the absorption band of ResOH, which exhibits a vibrational substructure with maxima at 305 and $318 \mathrm{~nm}$ in homogeneous aqueous solution, slightly towards longer wavelengths and significantly decreases its intensity. These changes (Fig. 2) clearly indicate complexation; to study
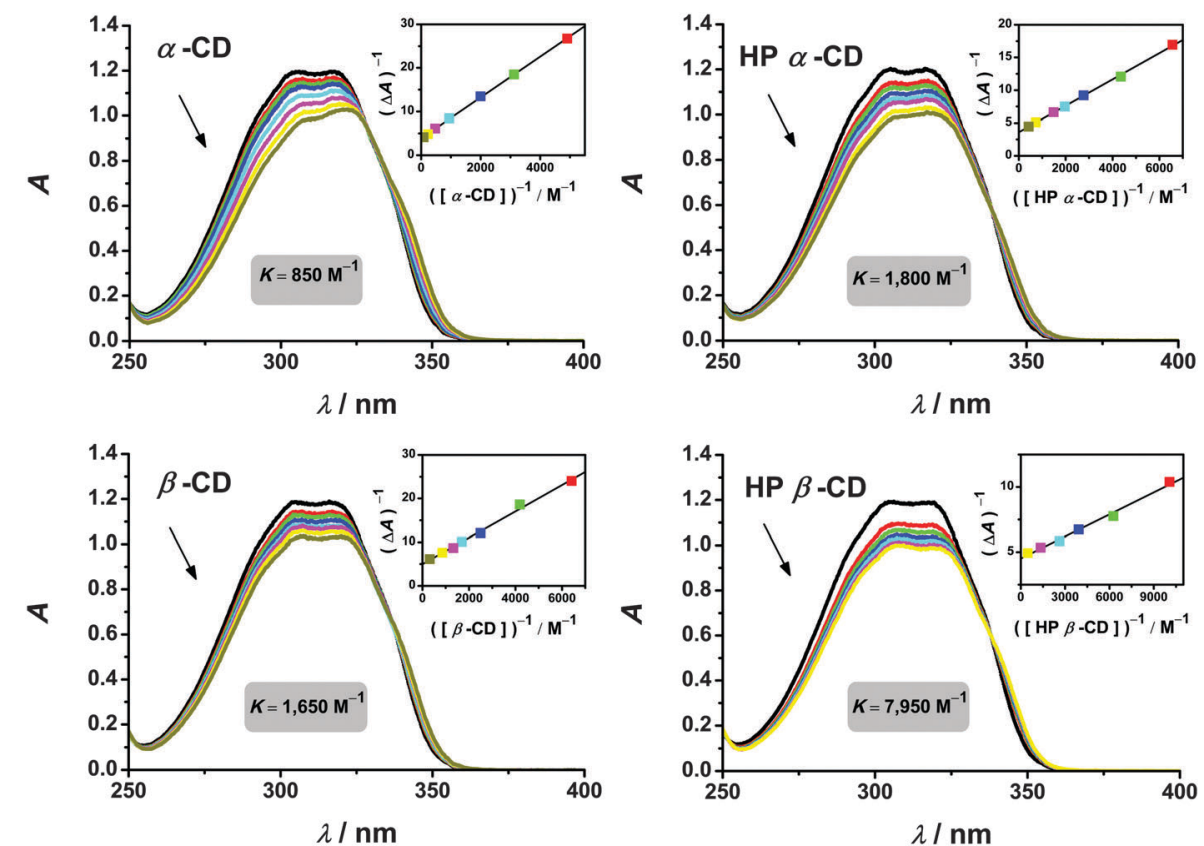

Fig. 2 Complexation of ResOH with cyclodextrins studied by UV absorption spectroscopy of aqueous solutions of $4 \times 10^{-5} \mathrm{M}$ ResOH at pH 6.5 and $297 \mathrm{~K}$ without (black spectra) and with varied amounts of the respective cyclodextrin (coloured spectra). The insets display the Benesi-Hildebrand plots of the absorbance changes $\Delta A$ using the same colour coding for the cyclodextrin concentrations as in the main plots. For further details, see the text. 
these equilibria quantitatively, we performed Benesi-Hildebrand analyses ${ }^{26}$ based on the absorptions. To that end, we recorded spectra with six to seven cyclodextrin concentrations distributed over an order of magnitude. For evaluation, we used the maxima in homogeneous solution that experience the largest absorption changes (318 nm, HP $\beta$-CD; $305 \mathrm{~nm}$, all other cyclodextrins).

In each case, a plot of the reciprocal absorbance change at the selected wavelength as a function of the reciprocal cyclodextrin concentration (Benesi-Hildebrand plot) gives a straight line with no discernible deviations (see the insets of Fig. 2). This identifies the complexation stoichiometry as $1: 1$, in agreement with previous studies of ResOH in cyclodextrins. ${ }^{4-12}$ The linear regressions have high correlation coefficients $\left(0.990<R^{2}<0.999\right)$ demonstrating that the UV absorption is well suited for investigating the cyclodextrin complexation of ResOH; their parameters yield the complexation constants, which are displayed in each respective subplot of Fig. 2 and also summarized in Table 1. These constants suggest two trends. First, the wider $\beta$-cyclodextrin cavity can accommodate ResOH in a less constrained manner than the tighter one of an $\alpha$-cyclodextrin; second, the extension of the effective cavity depth by HP substitution significantly helps binding this guest molecule.

2.1.2 Fluorescence. The reported fluorescence quantum yields of ResOH in homogeneous solution differed by one order of magnitude $\left(0.003^{27}\right.$ and $\left.0.023^{28}\right)$; hence we carefully re-determined this quantity against pyrene-1-sulfonate (PyS; fluorescence quantum yield, 0.71). ${ }^{29}$ The fluorescence spectrum of this standard overlaps well with that of ResOH (for comparison, see the inset of Fig. 3). Moreover, PyS is soluble in our reaction medium (water at $\mathrm{pH}$ 6.5), eliminating the influence of the refractive index.

Geometrical isomerization shortens the $\mathrm{S}_{1}$ lifetime of excited ResOH to $\sim 28 \mathrm{ps},{ }^{28,30}$ resulting in a very low fluorescence quantum yield. ${ }^{27,28}$ To compensate for the associated sensitivity loss, we increased the excitation slit of our spectrometer to $5 \mathrm{~nm}$, reduced the scan speed to $200 \mathrm{~nm}$ per minute, and averaged three independent measurements. Because we observed that a threefold repetition of the experiment on the

Table 1 Properties of $\mathrm{Res} O \mathrm{OH}$ and $\mathrm{Res}^{\bullet}$ encapsulated by cyclodextrins ${ }^{a}$

\begin{tabular}{|c|c|c|c|c|c|c|}
\hline Cyclodextrin & $K^{b} / \mathbf{M}^{-1}$ & $\Phi_{\mathrm{f}}^{c}$ & $\tau^{d} / \mathrm{ps}$ & $\mathrm{e}_{\mathrm{aq}}{ }^{\cdot-}$ yield $^{e}$ & $\begin{array}{l}\varepsilon_{410}\left(\operatorname{ResO}^{\bullet}\right) / \\
\left(\mathbf{M}^{-1} \mathrm{~cm}^{-1}\right)\end{array}$ & $k_{\text {repair }}$ \\
\hline- & - & 1 & 28 & 1 & 33400 & 1 \\
\hline$\alpha-C D$ & 850 & 4.5 & 160 & 3.1 & 21300 & 0.37 \\
\hline$\beta-\mathrm{CD}$ & 1650 & 2.1 & 70 & 2.3 & 22200 & 0.45 \\
\hline HP $\alpha$-CD & 1800 & 7.1 & 250 & 4.0 & 20200 & 0.25 \\
\hline HP $\beta$-CD & 7950 & 4.2 & 140 & 3.4 & 18300 & 0.27 \\
\hline
\end{tabular}

${ }^{a}$ At 297 K. ${ }^{b}$ Association constant of the $1: 1$ complex of ResOH with the respective cyclodextrin. ${ }^{c}$ Fluorescence quantum yield of ResOH relative to the value in homogeneous aqueous solution, 0.0039. ${ }^{d}$ Excitedstate lifetime of ResOH obtained by a Strickler-Berg analysis. ${ }^{e}$ Relative yield of $\mathrm{e}_{\mathrm{aq}}{ }^{--}$in the $355 \mathrm{~nm}$ photoionization of ResOH at $400 \mathrm{~mJ} \mathrm{~cm}^{-2}$ (corresponding to the highest laser intensity used). ${ }^{f}$ Rate constant for the repair reaction of $\mathbf{R e s O}^{\circ}$ by $\mathbf{H A s c}^{-}$(eqn (2)) with the value in homogeneous aqueous solution, $7.1 \times 10^{7} \mathrm{M}^{-1} \mathrm{~s}^{-1}$, as a reference.

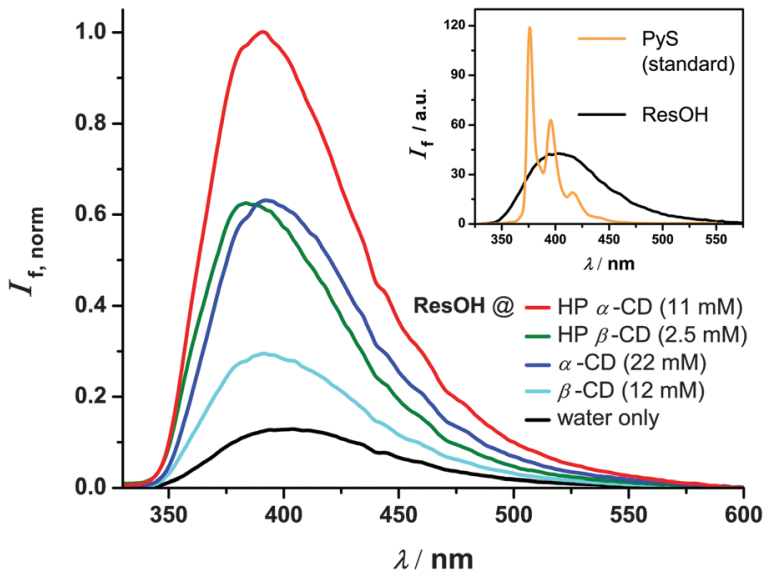

Fig. 3 Main plot: influence of the complexation with cyclodextrins on the fluorescence of an aqueous solution of $4 \times 10^{-5} \mathrm{M} \mathrm{ResOH}$ at $\mathrm{pH} 6.5$ and $297 \mathrm{~K}$. With the cyclodextrin concentrations given in the legend lines, $95 \%$ of ResOH is present in complexed form. All luminescence intensities $I_{\text {f,norm }}$ were acquired under identical excitation conditions and normalized to the maximum of the spectrum with HP $\alpha-C D$. Inset: comparison of the fluorescence spectra of ResOH and PyS, which was used as the fluorescence standard for uncomplexed ResOH in water. For further details, see the text.

same sample already led to a detectable presence of the unwanted cis isomer under these conditions, we used fresh solution for each of the three measurements.

By this methodology, we were further able to keep the absorbances of our samples below 0.15 to avoid inner filter effects, which are the most likely causes of the discrepancies reported in ref. 6 between the ResOH-cyclodextrin complexation constants determined by fluorescence in strongly absorbing solutions $(A \approx 0.6)$ and by other methods. Rather than using extremely small ResOH concentrations to that end, we excited in the vicinity of the minimum at the left edge of the spectra in Fig. 2. Excitation at either $250 \mathrm{~nm}$ or $260 \mathrm{~nm}$ gave very similar quantum yields $(0.0039 \pm 3 \%)$. The average value is in good agreement with that obtained by Deak et al. ${ }^{27}$

Next, we extended these investigations to the complexed molecule. A quantitative $(\geq 99 \%)$ complexation of $\mathbf{R e s O H}$ would require unreasonably high concentrations of our cyclodextrins (for $\beta$-CD even far above the solubility limit). Instead, we standardized all systems to $95 \%$ complexation, which requires the cyclodextrin concentrations listed in Fig. 3. The remaining contribution of free $\mathbf{R e s} \mathbf{O H}$ is easily removed by subtracting the fluorescence spectrum recorded in the absence of cyclodextrins after weighting it with a factor of 0.05 ; scaling the result with $1 / 0.95$ then yields the pure spectrum of complexed ResOH. Both corrections are seen to be so small as not to impede the reliability.

Furthermore, we excited at the isosbestic point of free ResOH and its respective complexed form $(250 \mathrm{~nm}$ for the two $\alpha$-cyclodextrins and $252 \mathrm{~nm}$ for the two $\beta$-cyclodextrins; see Fig. 2), where minute adjustments of the ResOH concentration sufficed to keep the absorbances of all solutions identical. As an advantage over previous investigations, ${ }^{6,8,11}$ the described 
procedure allows an immediate quantification of the cyclodextrin influences simply by comparing the fluorescence spectra.

The main plot of Fig. 3 collates the relative fluorescence spectra of differently complexed and uncomplexed ResOH. Whereas the spectral shape and the position of the maximum are hardly affected by the cyclodextrins, the emission becomes considerably stronger upon complexation. The fluorescence quantum yields $\Phi_{\mathrm{f}}$ obtained by integration are listed in Table 1 . They approximately double when the container is tightened (from a $\beta$-cyclodextrin to the $\alpha$-analogue) or deepened (from an unsubstituted cyclodextrin to its HP-substituted counterpart); a similar increase is observed when free $\mathbf{R e s} \mathrm{OH}$ becomes encapsulated by $\beta$-CD. It seems natural to ascribe these effects to an impeded twist of the resveratrol skeleton by the cyclodextrin cavity, which decelerates the radiationless deactivation through cis-trans isomerization, and thereby lengthens the life of the excited state and thus increases the emission probability.

The excited-state lifetime $\tau$ is the ratio of $\Phi_{\mathrm{f}}$ and the rate constant of fluorescence $k_{\mathrm{f}}$. The latter can in turn be calculated from the absorption and emission spectra and the refractive index $n$ using the well-known Strickler-Berg equation. ${ }^{31}$ For a vanishing Stokes shift and a mirror-image symmetry of the two spectra, this equation simplifies to a proportionality between $k_{\mathrm{f}}$ and the integrated absorption spectrum times $n^{2}$ times the squared energy of the $0-0$ transition. ${ }^{32}$ Although these conditions are approximately met by our systems, a much better reliability than for the absolute values of $k_{\mathrm{f}}$ is expected for the relative values, i.e., relative to the rate constant in homogeneous aqueous solution, $1.4 \times 10^{8} \mathrm{~s}^{-1}$, which directly follows from the known $\tau^{28,30}$ and our measured $\Phi_{\mathrm{f}}$. The lifetimes $\tau$ so obtained are included in Table 1 . Within $10 \%, \tau$ and $\Phi_{\mathrm{f}}$ show the same relationships with the cyclodextrin structures, which identifies the change in the oscillator strength of the transition as a minor factor; even the parameter $n^{2}$ remains constant within $1 \%$ when going from water to a cyclodextrin interior. ${ }^{32}$

The observed trend is in line with the reported quadrupling of the fluorescence lifetime of trans-stilbene, i.e., the structural parent of ResOH, upon complexation with $\alpha-\mathrm{CD} .{ }^{33}$ A further consistency test is provided by the successful fits of the photoionization yields with $\tau$ as fixed parameters (see the next section).

\subsection{Generation of the resveratrol radical}

Our recently developed strategy of photoionizing ResOH using a $355 \mathrm{~nm}$ laser pulse ${ }^{25}$ generates the $\mathbf{R e s O}^{\bullet}$ radical exactly at the location of its precursor, and therefore appears to be particularly promising for the cyclodextrin-complexed systems. We have already demonstrated the feasibility of this concept for $\mathbf{R e s O H}$ in anionic micelles. ${ }^{25}$ In the same way, an intense laser pulse on cyclodextrin-encapsulated ResOH brings about photoionization on a large scale. This is established by the characteristic absorption of $\mathrm{e}_{\mathrm{aq}}{ }^{\bullet-}$, which fades away within a few microseconds and can be removed completely by the selective electron scavenger $\mathrm{N}_{2} \mathrm{O}^{34}$ (Fig. 4a, inset).

The main plot of Fig. 4a displays the intensity dependence of the $\mathrm{e}_{\mathrm{aq}}{ }^{\bullet-}$ yield, relative to the initial ResOH concentration, in the four cyclodextrins investigated in this work and in homogeneous aqueous solution as a reference. Exploiting an enhanced sensitivity of our detection system at $824 \mathrm{~nm},{ }^{25}$ we observed $\mathrm{e}_{\mathrm{aq}}{ }^{\bullet-}$ at this wavelength and removed a very small contribution of $\mathbf{R e s O}^{\bullet}$ to the absorption signal by difference experiments, as shown in the inset of Fig. 4a. For the measurements on complexed ResOH, we again chose the cyclodextrin concentrations so as to bind $95 \%$ of our substrate
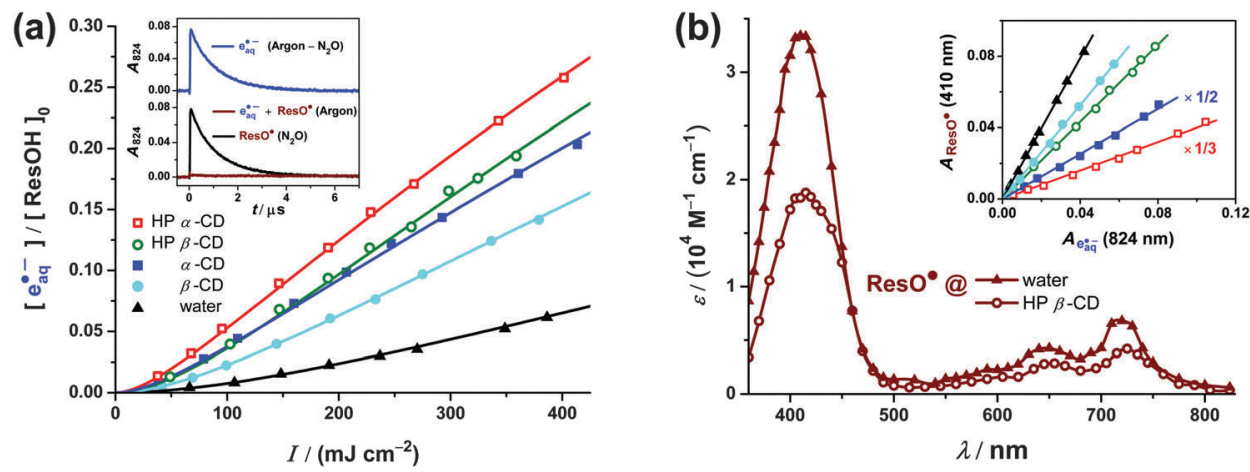

Fig. 4 Influence of cyclodextrins on the near-UV (355 nm) photoionization of an aqueous solution of $6 \times 10^{-5} \mathrm{M} \mathrm{ResOH}\left([\mathrm{ResOH}]_{0}\right)$ at pH 6.5 . (a) Main plot: intensity dependence of $\mathrm{e}_{\mathrm{aq}}{ }^{-}$formation in homogeneous aqueous solution (black data and fit function) and in the presence of cyclodextrins (coloured data and fit functions). Cyclodextrin concentrations are the same as in Fig. 3. Fit functions, $\left[\mathrm{e}_{\mathrm{aq}}{ }^{\bullet-}\right]=1-\exp \left(-\frac{a I^{2}}{1+B_{0} \varepsilon \tau I}\right)$. Global best-fit parameter $B_{0}, 5.91 \times 10^{4} \mathrm{~cm}^{3} \mathrm{M} \mathrm{mJ}^{-1} \mathrm{~s}^{-1}$; constants $\tau$ from Table 1 . Medium/constant parameters $\varepsilon$ (in $\left.\mathrm{M}^{-1} \mathrm{~cm}^{-1}\right) /$ best-fit parameters a (in $10^{6} \mathrm{~cm}^{4} \mathrm{~mJ}^{-2}$ ); water/800/0.65, $\alpha-\mathrm{CD} / 2100 / 12.3, \beta-\mathrm{CD} / 1440 / 3.52, \mathrm{HP} \alpha-\mathrm{CD} / 1720 / 21.0$, and HP $\beta-\mathrm{CD} / 1370 / 8.74$. Inset: representative absorption traces illustrating the separation of ResO ${ }^{\cdot}$ and $\mathrm{e}_{\mathrm{aq}}{ }^{-}$absorption signals by difference measurements in $\mathrm{Ar}$ - and $\mathrm{N}_{2} \mathrm{O}$-saturated solution with all other experimental parameters ( $2.5 \mathrm{mM} \mathrm{HP} \beta$-CD; laser intensity, $359 \mathrm{~mJ} \mathrm{~cm}{ }^{-2}$ ) unmodified. (b) Main plot: calibrated absorption spectrum of ResO• in water (filled triangles) and encapsulated by HP $\beta-\mathrm{CD}$ (open circles). Inset: ResO $0^{\bullet}$ absorptions at the spectral maximum (410 $\mathrm{nm}$ ) against the $\mathrm{e}_{\mathrm{aq}}{ }^{\bullet-}$ absorptions at a reference wavelength $(824 \mathrm{~nm}$ ) in homogeneous solution and in the presence of cyclodextrins at different laser intensities (same color coding as in the main plot of (a)); the respective slopes of the regression lines are used for calibration of the ResO• spectra (for clarity, two datasets have been rescaled). For further explanations, see the text. 
(for comparison, see Section 2.1.2 and Fig. 3). As we found from the difference spectra in Ar- and $\mathrm{N}_{2} \mathrm{O}$-saturated solutions, the broad band of $\mathrm{e}_{\mathrm{aq}}{ }^{\bullet-}$ peaking at about $720 \mathrm{~nm}$ in phosphatebuffered homogeneous aqueous solution ${ }^{25}$ remains unchanged and unshifted by the addition of millimolar concentrations of our cyclodextrins; hence, we were able to use our recently determined molar absorption coefficient $\left(\varepsilon_{824}\left(\mathrm{e}_{\mathrm{aq}}{ }^{\cdot-}\right)=16900 \mathrm{M}^{-1} \mathrm{~cm}^{-1}\right)^{25}$ for obtaining the $\mathrm{e}_{\mathrm{aq}}{ }^{\bullet-}$ concentrations.

The upward curvatures in the low-intensity regime and the negative intercepts of the straight-line extrapolations to zero intensity characterize the ionizations as biphotonic ${ }^{35}$ regardless of the environment of ResOH. However, the cyclodextrin complexation increases the $\mathrm{e}_{\mathrm{aq}}{ }^{\bullet-}$ yield from ResOH by a factor between 2 and 4, as is evidenced by the main plot of Fig. 4a. All dependence on the intensity $I$ can be fitted excellently with a two-parameter model, $\left[\mathrm{e}_{\mathrm{eq}}^{\bullet-}\right]=1-\exp \left(-\frac{a I^{2}}{1+b I}\right)$, which we have developed for the ionization of an excited state replenished by a fast photostationary pre-equilibrium, ${ }^{36}$ a condition that is almost perfectly met by ResOH owing to its short excited-state lifetime $\tau .{ }^{25}$ In addition, because of the composition of $b^{36}$ all five data sets can be fitted simultaneously with a global, medium-independent parameter $B_{0}$, from which each specific value of $b$ is derived by multiplication with the predetermined ground-state molar absorption coefficient $\varepsilon$ of ResOH at the excitation wavelength and with $\tau$, both in the respective environment. We regard the success of this fit-with $a$ as the only adjustable parameter for each data set-as incontrovertible evidence for the biphotonic ionization mechanism via the $\mathrm{S}_{1}$ state of ResOH ( $\left.{ }^{*} \mathbf{R e s O H}\right)$ according to ${ }^{25,28}$

$$
\operatorname{Res} \mathbf{O H} \underset{1 / \tau}{\stackrel{h \nu_{1}}{\rightleftharpoons}}{ }^{*} \operatorname{Res} \mathrm{OH} \stackrel{h \nu_{2}}{\longrightarrow} \mathrm{e}_{\mathrm{aq}}{ }^{\bullet-}\left(+\mathbf{R e s} \mathbf{O}^{\bullet}+\mathrm{H}^{+}\right)
$$

in the cyclodextrins as well. With the described model, the parameter ratio $a / b$ is proportional to the molar absorption coefficient of the $S_{1}$ state times the quantum yield of the ionization proper, and contains no other substrate- or medium-related quantities. ${ }^{36}$ Inspection of the fit results reveals this ratio to vary in parallel with the above-mentioned trends in $\tau$, but with a noticeably lower (by a factor of about 3) sensitivity to the environment. Because the ionization wavelength coincides with a spectral minimum of the $S_{1}$ state, ${ }^{28}$ the medium effects on the molar absorption coefficient should vanish to first order, and we ascribe this correlation to a common cause, namely, the competition of the observed process (above, luminescence; here, electron detachment) with radiationless deactivation, through geometrical isomerization, of the state from which the process occurs (above, $\mathrm{S}_{1}$; here, an upper excited state); the reason for the lower medium-influence on the electron ejection is a higher intrinsic rate of this process.

Fig. $4 \mathrm{~b}$ focuses on the absorption spectrum of ResO'. All these measurements were carried out in $\mathrm{N}_{2} \mathrm{O}$-saturated solution, where the strongly absorbing $\mathrm{e}_{\mathrm{aq}}{ }^{\bullet-}$ are instantaneously (i.e., within the duration of our laser pulses) converted into nonabsorbing hydroxyl radicals. The latter are in turn scavenged by the cyclodextrins to give carbon centered radicals, which are non-absorbing in our detection range; ${ }^{37}$ these diffusion controlled reactions are completed within nanoseconds at our cyclodextrin concentrations. On a longer timescale, these radicals are ultimately repaired by $\mathbf{R e s O H , ~ w h i c h ~ i s ~ a l s o ~ t h e ~ o n l y ~}$ sink of the hydroxyl radicals in the absence of cyclodextrins. However, at low ResOH concentrations used in our experiments, none of these secondary processes play any role for the concentrations of $\mathbf{R e s O}^{\bullet}$ immediately after the laser flash, on which Fig. $4 \mathrm{~b}$ is based.

The inset of Fig. $4 \mathrm{~b}$ compares the post-flash absorbances of ResO $^{\bullet}$ (at its absorption maximum, $410 \mathrm{~nm}$ ) and $\mathrm{e}_{\mathrm{aq}}{ }^{\bullet-}$ (without $\mathrm{N}_{2} \mathrm{O}$ and at its monitoring wavelength, $824 \mathrm{~nm}$, where ResO' is almost non-absorbing) throughout our range of laser intensities. The strict proportionality of the signals clearly indicates that both species, $\mathbf{R e s O}^{\bullet}$ and $\mathrm{e}_{\mathrm{aq}}{ }^{\bullet-}$, are produced by the same elementary reaction step not only in homogeneous solution, but also in the presence of cyclodextrins. The initial product of this photoionization is the resveratrol radical cation, which is known to undergo instantaneous deprotonation in aqueous and other homogeneous media to give $\mathbf{R e s O}^{\bullet}$ as the only observable resveratrol-derived radical form. ${ }^{25,28}$ Based on the absence of any additional signals, we conclude that this is case in the cyclodextrin systems as well. Hence, the absorption spectrum of cyclodextrin-complexed ResO ${ }^{\bullet}$ can be calibrated in a straightforward way against the $\mathrm{e}_{\mathrm{aq}}{ }^{\bullet-}$ absorption as the reference; to reduce the errors of the singlepoint measurements, we used the slopes of the regression lines in the inset of the figure. The molar absorption coefficients so obtained are compiled in Table 1.

The main plot of Fig. 4b finally juxtaposes the calibrated absorption spectra of $\mathbf{R e s O}^{\bullet}$ in homogeneous solution and complexed by HP $\beta$-CD, which we chose as an example because that cyclodextrin has the highest complexation constant for the starting material ResOH while for all four cyclodextrins investigated in this work the spectra of encapsulated $\mathbf{R e s O}^{\bullet}$ are very similar (see the molar absorption coefficients in Table 1). The complexation retains the spectral shape, with only slight red shifts of the maxima at about 410, 650 and $720 \mathrm{~nm}$, but decreases the molar absorption coefficients by about $40 \%$. This parallels the observations for ground-state ResOH (for comparison, see Fig. 2 and ref. 11) with an even more pronounced hypochromic effect, and therefore clearly indicates a complexation of $\mathbf{R e s O}^{\bullet}$ by all cyclodextrins used in this study.

In the presence of cyclodextrins, the described photoionization approach has a double preference for generating $\mathbf{R e s O}^{\bullet}$ in encapsulated form: (i) because of the complexation of the parent molecule ResOH combined with the fact that the radical is born at the very location of its precursor and (ii) because the photoionization of complexed ResOH is more efficient than that of free ResOH.

\subsection{Repair reaction of the resveratrol radical by ascorbate}

So far, the photoionization approach to cyclodextrin-encapsulated phenoxy radicals has been limited to the use of UV-C light (266 nm), and the few available reports have only been concerned with the generation of the radicals ${ }^{38,39}$ or their reaction with 
the container. ${ }^{40}$ More directly related to the present work are two studies on how the co-antioxidant HAsc ${ }^{-}$interacts with $\alpha$-tocopherol phenoxy radicals prepared by UV-B (308 $\mathrm{nm}$ ) photoionization in model membranes. ${ }^{19,20}$

For all experiments addressing the repair reaction (eqn (2)),

$$
\mathrm{ResO}^{\bullet}+\mathrm{HAsc}^{-} \rightarrow \operatorname{ResOH}+\mathrm{Asc}^{\bullet-}
$$

we generated ResO $^{\bullet}$ concentrations of about $5 \times 10^{-6} \mathrm{M}$ by the described near-UV ionization of ResOH, corresponding to some $10 \%$ conversion of this substrate (Fig. 4a).

Under these conditions, $\mathbf{R e s O}^{\bullet}$ alone disappears only on a timescale as long as hundreds of microseconds in homogeneous aqueous solution, ${ }^{25}$ and even more slowly in the presence of our cyclodextrins. While the complexity of this decay ${ }^{41}$ prohibited its use for studying the association/dissociation equilibrium between $\mathbf{R e s O}^{\bullet}$ and the cyclodextrins, its low rate permitted the undisturbed observation of the repair by ascorbate.

To eliminate the intense electron absorption, we worked with $\mathrm{N}_{2} \mathrm{O}$-saturated solutions (see Section 2.2), thereby instantaneously and quantitatively transforming $\mathrm{e}_{\mathrm{aq}}{ }^{\bullet-}$ into the non-absorbing hydroxyl radicals. As opposed to Section 2.2, in our experiments with HAsc $^{-}$(concentrations, several $\mathrm{mM}$ ) a significant amount of these hydroxyl radicals is already scavenged directly by $\mathbf{H A s c}^{-}$; the cyclodextrin-based radicals are also repaired by $\mathbf{H A s c}^{-}$; and the secondary formation of $\mathbf{R e s O}^{\bullet}$ is totally suppressed. Hence, all absorptions above $500 \mathrm{~nm}$ arise solely from ResO` produced by the photoionization flash. For selectively monitoring its further fate, we used its red absorption band, averaging the two maxima at 650 and $720 \mathrm{~nm}$ to improve the signal-to-noise ratio.

2.3.1 Kinetic investigations. Fig. 5 displays normalized experimental traces of $\mathbf{R e s}^{\bullet}$, both in homogeneous solution

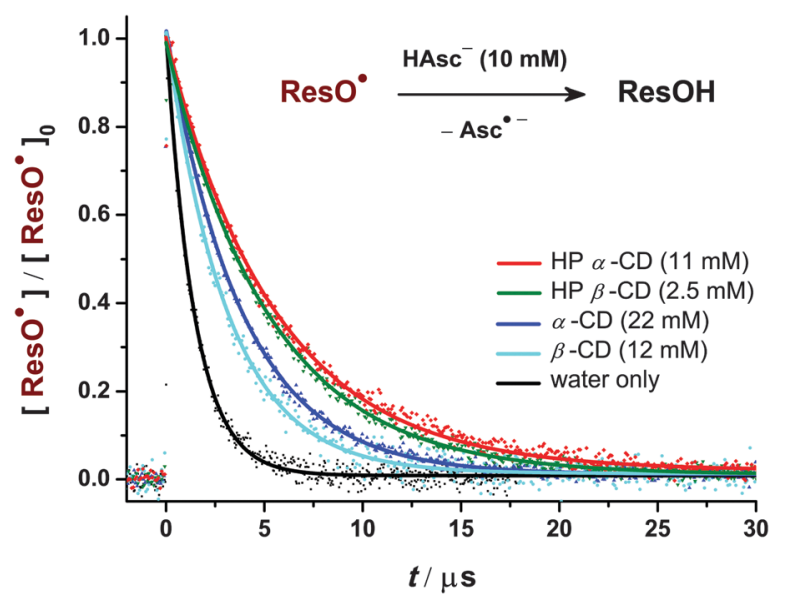

Fig. 5 Repair reaction of ResO by $10 \mathrm{mM} \mathrm{HAsc}^{-}$at $\mathrm{pH} 6.5$ and $297 \mathrm{~K}$ in homogeneous aqueous solution (black experimental data and fit function) and at the cyclodextrin-water interface (coloured experimental data and fit functions; cyclodextrin concentrations given in the legend lines). ResO' was selectively generated by near-UV photoionization of an $\mathrm{N}_{2} \mathrm{O}$ saturated solution of $\operatorname{ResOH}\left(6 \times 10^{-5} \mathrm{M}\right)$. The laser intensities were adjusted so as to convert about $5 \times 10^{-6} \mathrm{M}$ of ResOH into ResO'. The experimental data were normalized to the initial radical concentration $\left[\mathrm{Res}^{*}\right]_{0}$ and fitted with the functions $\exp \left(-k_{\text {obs }} t\right)$. For further information, see the text. and complexed by the cyclodextrins used in this study, at the same HAsc $^{-}$concentration (2000-fold excess over ResO`). In all cases, $\mathbf{R e s O}^{\bullet}$ completely vanishes within a few tens of microseconds at most, and the decay is perfectly monoexponential, indicating first-order kinetics with respect to the radical. It is evident that the repair according to eqn (2) is considerably slower in the presence of any of the cyclodextrins at its standard concentration (corresponding to $95 \%$ complexation of the starting material ResOH, as discussed in Section 2.1.2).

Because the laser flash preferentially ionizes ResOH residing within a cyclodextrin (see Section 2.2) and generates the radical $\mathbf{R e s O}^{\bullet}$ at the location of that precursor, the initial post-flash fraction of complexed ResO' exceeds that of complexed $\mathbf{R e s O H}$ and lies between 98\% ( $\beta$-CD) and 99\% (HP $\alpha$-CD). The differences between the curves of Fig. 5 could thus be caused by a continuum of possibilities between two extreme situations that (a) the repair reaction according to eqn (2) occurs not only in homogeneous solution but also-with a substantial deceleration-at the cyclodextrin-water interface, and the exchange of ResO' between cyclodextrin and water is too slow to play any role; or (b) the repair is only possible in the aqueous phase while the decomplexation of $\mathbf{R e s O}^{\bullet}$ is rate-limiting.

To determine the position within this mechanistic spectrum, we first varied the HAsc ${ }^{-}$concentration between 5 and $25 \mathrm{mM}$. In the two limiting cases, the observed rate constant $k_{\text {obs }}$ would have to be concentration-proportional (a) or completely concentrationindependent (b); in-between, one would expect some non-linear concentration dependence. All the plots of $k_{\mathrm{obs}}$ as a function of [HAsc ${ }^{-}$] were linear with negligible intercepts (an example is given in the inset of Fig. 6a), establishing case (a) as well as confirming that radical-radical recombinations are unimportant under our conditions.

For further corroboration, we also carried out experiments at constant $\left[\mathbf{H A s c}^{-}\right]$but with lower cyclodextrin concentrations, that is, with a significant fraction of $\mathbf{R e s O}^{\bullet}$ present in uncomplexed form. Case (a) demands independent decays of complexed (index c) and free (index f) ResO ${ }^{\bullet}$ with their respective pseudo first-order rate constants, i.e., an integrated rate law $n_{\mathrm{c}} \exp \left(-k_{\mathrm{c}} t\right)+n_{\mathrm{f}}$ $\exp \left(-k_{\mathrm{f}} t\right)$, with the rate constants $k_{\mathrm{c}}$ and $k_{\mathrm{f}}$ and the initial mole fractions $n_{\mathrm{c}}$ and $n_{\mathrm{f}}$. The decays observed in these experiments were perfectly reproducible by this superposition, with $k_{\mathrm{c}}$ approximated by the rate constants obtained at twice the cyclodextrin concentrations given in Fig. 5 (except for $\beta$-CD, where the solubility limit allowed an increase by a factor of 1.5 only) and $k_{\mathrm{f}}$ measured without cyclodextrin, and with the mole fractions calculated from the results of Sections 2.1 and 2.2.

Another mechanistic complication would arise from binding of HAsc $^{-}$to the cyclodextrins. In one report, an association constant between HAsc $^{-}$and $\alpha$-CD of $112 \mathrm{M}^{-1}$ was inferred indirectly from kinetic measurements in a multicomponent system. $^{42}$ Because we considered such a complexation unlikely, given the less polar interior of the cyclodextrin and the delocalized negative charge of the guest, ${ }^{43}$ we recorded absorption spectra of HAsc $^{-}$in the presence of different cyclodextrin concentrations. We observed no changes even with $20 \mathrm{mM} \alpha$-CD, where the above equilibrium constant would predict $69 \%$ of HAsc $^{-}$to be 

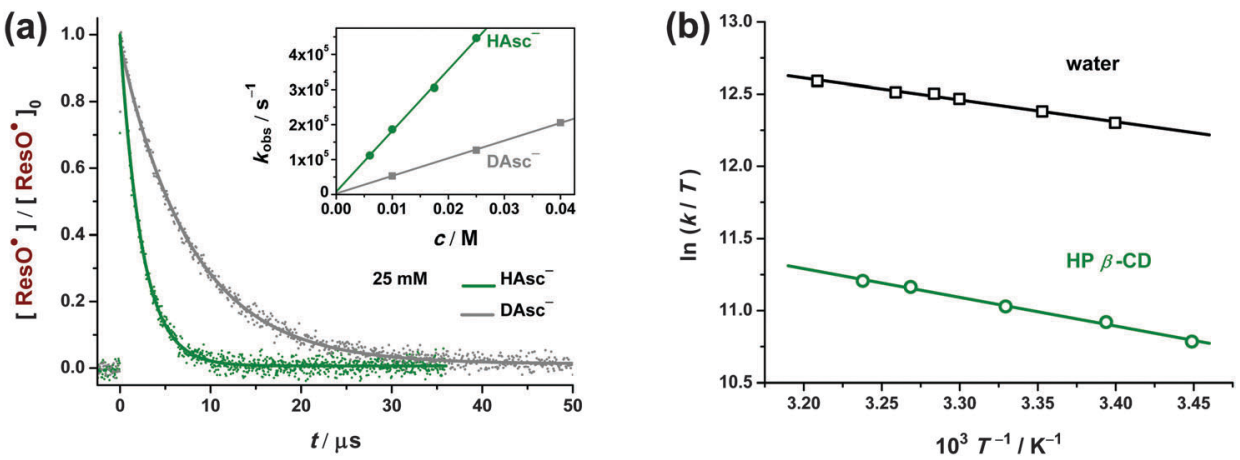

Fig. 6 Mechanistic studies on the repair of ResO• by ascorbate at the HP $\beta$-CD water interface and in homogeneous aqueous solution (black). (a) Kinetic isotope effect in $2.5 \mathrm{mM} \mathrm{HP} \beta-\mathrm{CD}$ at $297 \mathrm{~K}$. Main plot: normalized experimental decay traces for ResO' repaired by $25 \mathrm{mM}$ of protiated (green) or deuterated (gray) ascorbate overlaid with the best-fit functions $\exp \left(-k_{\mathrm{obs}} t\right)$. Inset: dependence of $k_{\text {obs }}$ (extracted from the fits for a series of measurements as in the main plot) on the ascorbate concentrations. (b) Eyring plots for the repair of complexed (green; $7.5 \mathrm{mM} \mathrm{HP} \beta$-CD; $20 \mathrm{mM}$ HAsc $^{-}$) and uncomplexed ResO• (black; $5 \mathrm{mM} \mathrm{HAsc}^{-}$). For further explanation, see the text.

encapsulated. In contrast, a control experiment in methanol/ water $(10: 1, \mathrm{v} / \mathrm{v})$, i.e., in a medium of comparable polarity to that presumed for the cyclodextrin interior, ${ }^{44}$ gave an unambiguous red shift of the narrow absorption band by $\sim 4 \mathrm{~nm}$. These findings rule out a noticeable degree of HAsc ${ }^{-}$ complexation in the measurements of this work.

From the experiments of this section, we can thus conclude that the cyclodextrin-water interface substantially decelerates the repair reaction: the second-order rate constants ( $k_{\text {repair }}$, which follow from the fit parameter $k_{\text {obs }}$ and the HAsc ${ }^{-}$ concentration, decrease by a factor of $2-4$, depending on the cyclodextrin (see Table 1).

2.3.2 Mechanistic investigations. The direct kinetic measurements of the preceding section have established that cyclodextrin encapsulation invariably decreases the repair rate of ResO'. This unambiguous result contrasts markedly with the contradictory information about the influence of cyclodextrins on radical scavenging by complexed ResOH, which is the formal back reaction of eqn (2), hence strongly related to the ResO• repair. This antioxidative action of ResOH encapsulated by cyclodextrins has been addressed experimentally using activity assays ${ }^{7,9,15,16}$ and kinetic measurements. ${ }^{17}$ Whereas the DPPH assay showed no influence of the complexation on the scavenging, ${ }^{7,9}$ the ORAC assay in the presence of cyclodextrins suggested an improvement by $80 \% .{ }^{15,16}$ However, the prolonged activity in the latter case might simply reflect the increased stability of complexed ResOH towards air and light ${ }^{4,16}$ rather than a change in the rate constant of the scavenging proper, ${ }^{45,46}$ that is, of the parameter characterizing the antioxidant activity at a molecular level. The only available kinetic study supports a quite low influence of cyclodextrins on radical scavenging by ResOH: even with a methylated cyclodextrin, which is capable of covering a larger part of ResOH than an unmodified cyclodextrin, the rate constant of alkoxy radical scavenging decreased only by about $25 \%$ compared to that in homogeneous solution. ${ }^{17}$

The obviously stronger cyclodextrin effects on ResO' scavenging by the co-antioxidant $\mathbf{H A s c}^{-}$compared to radical scavenging by the antioxidant ResOH prompted us to check whether the repair according to eqn (2) undergoes a mechanistic change when ResO $\mathbf{O}^{\bullet}$ is complexed. Thermodynamically, a concerted process and a sequence of rate-determining electron transfer followed by fast proton transfer are feasible for the protoncoupled electron transfer (for details of this class of reactions, see ref. 47 and 48) of reaction (2).

Our recent study ${ }^{25}$ on the H/D kinetic isotope effect (KIE) of this reaction in homogeneous solution gave a KIE of 3.9, consistent with the one-step process. To extend this to cyclodextrin-complexed ResO', we chose HP $\beta$-CD because this host exhibits the strongest complexation of $\mathbf{R e s O H}$, the largest effect on the ResO' absorption and almost the maximum reduction of $k_{\text {repair }}$, making it the most likely of the four cyclodextrins for a mechanistic turnaround, if the latter were responsible for the observed rate decrease. Fig. 6a compares the ResO' repair kinetics in $\mathrm{H}_{2} \mathrm{O}$ and $\mathrm{D}_{2} \mathrm{O}$ (where all hydroxylic protons in the reactants are replaced by deuterons) with all other experimental parameters identical. The KIE obtained from Fig. 6a, 3.5, is very similar to the KIE that we measured in homogeneous aqueous solution, 3.9, ${ }^{25}$ indicating that the repair mechanism does not deviate from a one-step process upon cyclodextrin complexation of ResO'.

These findings narrow down the mechanistic possibilities to a hydrogen atom transfer (HAT) or a concerted proton-electron transfer (CPET). Both convert the linear fragment $\mathrm{O} \cdots \mathrm{H}-\mathrm{O}$ into $\mathrm{O}-\mathrm{H} \cdots \mathrm{O}$, yet with different orbital participation. The hydrogen atom (HAT) or the proton (CPET) travel along the $\sigma$ orbitals of the old and new bond, but the CPET additionally relies on a $\pi$ type overlap of the heteroatom lone pairs for the simultaneous shift of the electron. ${ }^{48,49}$ This discrepancy of geometric constraints is tantamount to retaining or losing the free rotation around the $\sigma$ bonds, and therefore must be reflected by a zero (HAT) or a strongly negative (CPET) contribution to the activation entropy. In both cases, this is overlaid with the same, small and positive, vibrational contribution arising mainly from the frequency differences between the $\mathrm{H}-\mathrm{O}$ stretching mode in the hydrogen-bonded starting state and the symmetrical $\mathrm{O} \cdots \mathrm{H} \cdots \mathrm{O}$ stretching mode in the activated complex, such that in total a small positive activation entropy results for HAT and a significantly negative one for CPET. ${ }^{20}$ 
Table 2 Mechanistic studies on the repair of ResO by ascorbate

\begin{tabular}{lllll}
\hline Medium & $\mathrm{KIE}^{a}$ & $\Delta H^{\ddagger b} /\left(\mathrm{kJ} \mathrm{mol}^{-1}\right)$ & $\Delta S^{\ddagger b} /\left(\mathrm{J} \mathrm{K}^{-1} \mathrm{~mol}^{-1}\right)$ & $\Delta G^{\ddagger c} /\left(\mathrm{kJ} \mathrm{mol}^{-1}\right)$ \\
\hline Water & $3.9^{25}$ & 12.6 & -52.2 & 28.2 \\
HP $\beta$-CD & 3.5 & 19.1 & -50.6 & 31.7
\end{tabular}

${ }^{a}$ H/D kinetic isotope effect. ${ }^{b}$ Obtained from the fit parameters of the regression lines displayed in Fig. $6 \mathrm{~b} .{ }^{c}$ Calculated at $298 \mathrm{~K}$.

To obtain the activation parameters, we carried out temperature dependent measurements of the $\mathbf{R e s O}^{\bullet}$ repair rate both in homogeneous aqueous solution and at the cyclodextrin-water interface (Fig. 6b). For this purpose, HP $\beta$-CD is again the most suitable of our cyclodextrins because its room-temperature complexation constant is already the largest by far, yet decreases only by $20 \%$ over the temperature interval of the experiments, ${ }^{8}$ as opposed to a factor of 2 for unsubstituted $\beta-C D,{ }^{5}$ and its high water solubility allowed a trebling of its concentration, thereby ensuring quantitative (>99\%) complexation of $\mathbf{R e s O}^{\bullet}$ at all temperatures.

The Eyring plots of Fig. $6 \mathrm{~b}$ are linear in both media. The activation parameters were calculated from the regression lines and are summarized in Table 2 . The negative activation entropies $\Delta S^{\ddagger}$ are consistent with the CPET process for reaction (2). In any case, the numerically and extremely similar (to within $3 \%$ ) values of $\Delta S^{\ddagger}$ clearly establish that the complexation does not entail a mechanistic change, whereas the appreciable difference in the activation enthalpies $\Delta H^{\ddagger}$ points to the steric repulsion $^{23}$ between the cyclodextrin and (non-complexable) HAsc $^{-}$as the reason for the deceleration by the macrocycle.

Different localizations of $\mathbf{R e s O H}$ and its phenoxy radical $\mathbf{R e s O}^{\bullet}$ within the containers provide a natural rationalization of the disparate kinetic effects. For ResOH, NMR experiments ${ }^{4}$ have shown the centre of its olefinic bond approximately to coincide with the centre of the cyclodextrin, regardless of whether the latter is native or HP-substituted. In consequence, the reactive single phenolic group extends sufficiently far into the aqueous bulk so as to allow almost unrestricted scavenging of radicals ${ }^{\circ} \mathrm{OR}$ (Fig. 7a). However, once this group has been converted into a phenoxy radical, which is significantly less hydrophilic than is $-\mathrm{OH}$ because of its inability to function as a hydrogen-bond donor, ${ }^{50}$ the guest $\mathbf{R e s O}^{\bullet}$ is drawn deeper into the hydrophobic interior of the host such that the access of the co-antioxidant HAsc $^{-}$is hindered by its interactions with the cyclodextrin rim (Fig. 7b). This model also explains why a $\beta$-cyclodextrin with its wider diameter causes only a slightly smaller deceleration of the $\mathbf{R e s O}^{\bullet}$ repair than does the corresponding $\alpha$-cyclodextrin whereas HP substitution, i.e., a dilation of the container in the direction of the long axis of $\mathbf{R e s O}^{\bullet}$, has a significantly larger effect.

\section{Experimental section}

All chemicals were obtained commercially in the highest available purity and used as received (trans-resveratrol, >98\%, TCI; sodium ascorbate, $\geq 99 \%$, Roth; sodium hydrogen phosphate, $\geq 99 \%$, Fluka; sodium dihydrogen phosphate, $>98 \%$, VEB Laborchemie Apolda; $\alpha$-cyclodextrin, >98\%, TCI; $\beta$-cyclodextrin, $>99 \%$, TCI; (2-hydroxypropyl)- $\beta$-cyclodextrin, molecular weight $1460 \mathrm{~g} \mathrm{~mol}^{-1}$, Aldrich; (2-hydroxypropyl)- $\alpha$-cyclodextrin, molecular weight $1180 \mathrm{~g} \mathrm{~mol}^{-1}$, Aldrich; sodium pyrene-1-sulfonate, $\geq 97 \%$, Aldrich; 2-aminopyridine, $\geq 99 \%$, Aldrich; $N, N$-dimethylamino- $m$ nitrobenzene, 98\%, Alfa Aesar; methanol, $\geq 99.9 \%$, Aldrich).

The solvent was either ultrapure Millipore Milli-Q water (specific resistance, $18.2 \mathrm{M} \Omega \mathrm{cm}$ ) or $\mathrm{D}_{2} \mathrm{O}(99.9 \%$ deuteration, Deutero $\mathrm{GmbH}$ ). The ResOH-containing solutions were always freshly prepared before experiments and stored in the dark to avoid degradation. To ensure the presence of (fully protonated) ResOH only, all measurements were carried out at $\mathrm{pH} 6.5,{ }^{25}$ using a phosphate buffer (general buffer concentration, $6.7 \mathrm{mM}$ ) prepared by mixing stock solutions of sodium hydrogen phosphate and sodium dihydrogen phosphate.

Steady-state absorption and fluorescence spectra were recorded using a Shimadzu UV-2102 spectrophotometer and a Perkin Elmer LS 50B spectrometer. Control experiments without ResOH established the absence of absorption or luminescence signals from the cyclodextrins. The fluorescence quantum yields were determined in deaerated solutions to avoid oxygen quenching of the excited standard pyrene-1-sulfonate ${ }^{29}$ (the fluorescence of ResOH is insensitive to oxygen). The fluorescence spectrometer was calibrated with the standards 2-aminopyridine and (a)

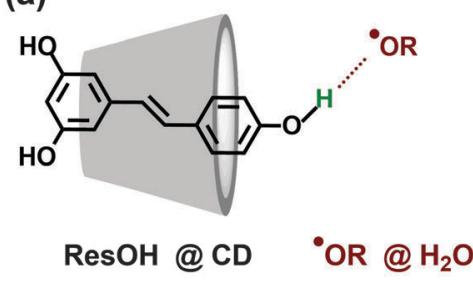

(b)

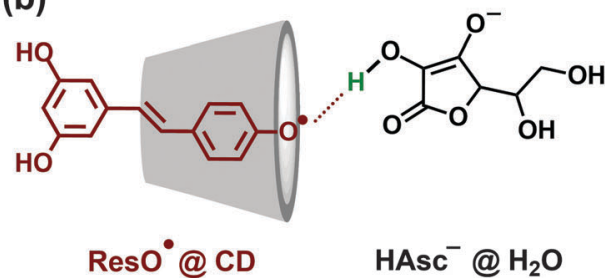

decelerated repair reaction

\section{unhindered radical scavenging}

Fig. 7 Structures of the complexed species explaining the observed effects of cyclodextrins on radical scavenging by ResOH and on the repair of ResO•. (a) Confirmed structure ${ }^{4}$ of the ResOH cyclodextrin complex (ResOH(aCD) allowing unhindered radical scavenging. (b) Most likely structure, owing to a higher guest hydrophobicity of complexed ResO• (ResO $(\mathrm{aCD})$, in which the phenoxy group is shielded by the cyclodextrin cavity, impeding access of co-antioxidant $\mathrm{HAsc}^{-}$from the aqueous phase. For further details, see the text. 
$N, N$-dimethylamino- $m$-nitrobenzene. ${ }^{51}$ The sensitivity factors so obtained were used to correct all emission spectra.

The laser flash photolysis setup featured a frequency-tripled Nd:YAG laser (Continuum Surelite-III, $355 \mathrm{~nm}, 5 \mathrm{~ns}$ pulse width) for excitation and optical detection of the intermediates. A detailed description has been given elsewhere. ${ }^{52}$ All solutions were purged with argon (5.0, Air Liquide) or $\mathrm{N}_{2} \mathrm{O}$ (5.0, Air Liquide) for 30 minutes before and for the whole duration of the laser flash photolysis experiments. To avoid the oxidation of the co-antioxidant ascorbate by molecular oxygen, ${ }^{53}$ the required amount of sodium ascorbate was added in solid form to the already degassed solutions immediately before starting the laser experiments.

Unless otherwise noted, all experiments were carried out at room temperature $(297 \pm 1 \mathrm{~K})$.

\section{Conclusions}

These experiments have demonstrated the wealth of information that is accessible by the selective in situ generation of the resveratrol phenoxy radical at the precise location of its parent compound, such as within a cyclodextrin cavity, through UV-A photoionization. Because no auxiliary reagent is involved in its production, the repair reaction by ascorbate-a key step for the bioavailability of the famous antioxidant resveratrol-can be observed in isolation, providing unequivocal answers about the factors that govern it. This approach clearly paves the way for investigations of the interactions between other antioxidants across the phase boundaries of microheterogeneous systems.

\section{Acknowledgements}

Financial support provided by the Fonds der Chemischen Industrie to C. K. (PhD scholarship) is gratefully acknowledged.

\section{References}

1 B. B. Aggarwal and S. Shishodia, Resveratrol in health and disease, CRC Press, Boca Raton, 1st edn, 2005.

2 P. Farris, J. Krutmann, Y.-H. Li, D. McDaniel and Y. Krol, J. Drugs Dermatol., 2013, 12, 1389-1394.

3 B.-Y. Chen, C.-H. Kuo, Y.-C. Liu, L.-Y. Ye, J.-H. Chen and C.-J. Shieh, J. Nat. Prod., 2012, 75, 1810-1813.

4 V. Bertacche, N. Lorenzi, D. Nava, E. Pini and C. Sinico, J. Inclusion Phenom. Macrocyclic Chem., 2006, 55, 279-287.

5 J. M. López-Nicolás and F. García-Carmona, Food Chem., 2008, 109, 868-875.

6 C. Lucas-Abellán, M. I. Fortea, J. A. Gabaldón and E. NúñezDelicado, Food Chem., 2008, 111, 262-267.

7 Z. Lu, B. Cheng, Y. Hu, Y. Zhang and G. Zou, Food Chem., 2009, 113, 17-20.

8 Z. Lu, R. Chen, H. Liu, Y. Hu, B. Cheng and G. Zou, J. Inclusion Phenom. Macrocyclic Chem., 2009, 63, 295-300.

9 S. Sapino, M. E. Carlotti, G. Caron, E. Ugazio and R. Cavalli, J. Inclusion Phenom. Macrocyclic Chem., 2009, 63, 171-180.
10 H. Li, X. Xu, M. Liu, D. Sun and L. Li, Thermochim. Acta, 2010, 510, 168-172.

11 J. M. López-Nicolás and F. García-Carmona, Food Chem., 2010, 118, 648-655.

12 E. Troche-Pesqueira, I. Pérez-Juste, A. Navarro-Vázquez and M. M. Cid, RSC Adv., 2013, 3, 10242-10250.

13 J. Chaudière and R. Ferrari-Iliou, Food Chem. Toxicol., 1999, 37, 949-962.

14 J. E. Packer, T. F. Slater and R. L. Wilson, Nature, 1979, 278, 737-738.

15 C. Lucas-Abellán, M. T. Mercader-Ros, M. P. Zafrilla, M. I. Fortea, J. A. Gabaldón and E. Núñez-Delicado, J. Agric. Food Chem., 2008, 56, 2254-2259.

16 C. Lucas-Abellán, M. T. Mercader-Ros, M. P. Zafrilla, J. A. Gabaldón and E. Núñez-Delicado, Food Chem. Toxicol., 2011, 49, 1255-1260.

17 M. Ishikawa, Y. Sueishi, N. Endo, S. Oowada, M. Shimmei, H. Fujii and Y. Kotake, Int. J. Chem. Kinet., 2012, 44, 598-603.

18 E. Niki, J. Tsuchiya, R. Tanimura and Y. Kamiya, Chem. Lett., 1982, 789-792.

19 R. H. Bisby and A. W. Parker, FEBS Lett., 1991, 290, 205-208.

20 R. H. Bisby and A. W. Parker, Arch. Biochem. Biophys., 1995, 317, 170-178.

21 W. Gregor, G. Grabner, C. Adelwöhrer, T. Rosenau and L. Gille, J. Org. Chem., 2005, 70, 3472-3483.

22 K. Mukai, S. Mitani, K. Ohara and S. Nagaoka, Free Radical Biol. Med., 2005, 38, 1243-1256.

23 S. Nagaoka, T. Kakiuchi, K. Ohara and K. Mukai, Chem. Phys. Lipids, 2007, 146, 26-32.

24 J.-G. Fang, M. Lu, Z.-H. Chen, H.-H. Zhu, Y. Li, L. Yang, L.-M. Wu and Z.-L. Liu, Chem. - Eur. J., 2002, 8, 4191-4198.

25 C. Kerzig, S. Henkel and M. Goez, Phys. Chem. Chem. Phys., 2015, 17, 13915-13920.

26 H. A. Benesi and J. H. Hildebrand, J. Am. Chem. Soc., 1949, 71, 2703-2707.

27 M. Deak and H. Falk, Monatsh. Chem., 2003, 134, 883-888.

28 I. Džeba, T. Pedzinski and B. Mihaljević, J. Photochem. Photobiol., A, 2015, 299, 118-124.

29 C. Bohne, E. B. Albuin and J. C. Scaiano, J. Am. Chem. Soc., 1990, 112, 4226-4231.

30 R. Simkovitch and D. Huppert, J. Phys. Chem. B, 2015, 119, 11684-11694.

31 S. J. Strickler and R. A. Berg, J. Chem. Phys., 1962, 37, 814-822.

32 J. Mohanty and W. M. Nau, Photochem. Photobiol. Sci., 2004, 3, 1026-1031.

33 G. L. Duveneck, E. V. Sitzmann, K. B. Eisenthal and N. J. Turro, J. Phys. Chem., 1989, 93, 7166-7170.

34 J. W. T. Spinks and R. J. Woods, An Introduction to Radiation Chemistry, Wiley, New York, 2nd edn, 1976.

35 U. Lachish, A. Shafferman and G. Stein, J. Chem. Phys., 1976, 64, 4205-4211.

36 M. Goez and C. Kerzig, Angew. Chem., Int. Ed., 2012, 51, 12606-12608.

37 D. La Mendola, S. Sortino, G. Vecchio and E. Rizzarelli, Helv. Chim. Acta, 2002, 85, 1633-1643. 
38 I. P. Pozdnyakov, L. Guo, E. M. Glebov, F. Wu, V. F. Plyusnin, V. P. Grivin and N. Deng, High Energy Chem., 2011, 45, 214-221.

39 V. A. Salomatova, I. P. Pozdnyakov, V. V. Yanshole, F. Wu, V. P. Grivin, N. M. Bazhin and V. F. Plyusnin, J. Photochem. Photobiol., A, 2014, 274, 27-32.

40 S. Monti and S. Sortino, Chem. Soc. Rev., 2002, 31, 287-300.

41 S. Stojanović and O. Brede, Phys. Chem. Chem. Phys., 2002, 4, 757-764.

42 Y. L. Loukas, V. Vraka and G. Gregoriadis, Pharm. Sci., 1996, 2, 523-527.

43 J. W. Park, Fluorescence Methods for Studies of Cyclodextrin Inclusion Complexation and Excitation Transfer in Cyclodextrin Complexes, in Cyclodextrin Materials Photochemistry, Photophysics and Photobiology, ed. A. Douhal, Elsevier, Amsterdam, 2006, pp. 1-26.

44 B. D. Wagner, The Effects of Cyclodextrins on Guest Fluorescence, in Cyclodextrin Materials Photochemistry, Photophysics and Photobiology, ed. A. Douhal, Elsevier, Amsterdam, 2006, pp. 27-59.

45 E. Niki, Free Radical Biol. Med., 2010, 49, 503-515.
46 Y. Takahashi, K. Ichimori, M. Okano and H. Goto, J. Clin. Biochem. Nutr., 2015, 56, 105-110.

47 D. R. Weinberg, C. J. Gagliardi, J. F. Hull, C. F. Murphy, C. A. Kent, B. C. Westlake, A. Paul, D. H. Ess, D. G. McCafferty and T. J. Meyer, Chem. Rev., 2012, 112, 4016-4093.

48 J. J. Warren, T. A. Tronic and J. M. Mayer, Chem. Rev., 2010, 110, 6961-7001.

49 J. M. Mayer, D. A. Hrovat, J. L. Thomas and W. T. Borden, J. Am. Chem. Soc., 2002, 124, 11142-11147.

50 R. C. Guedes, K. Coutinho, B. J. Costa Cabral and S. Canuto, J. Phys. Chem. B, 2003, 107, 4304-4310.

51 K. Suzuki, A. Kobayashi, S. Kaneko, K. Takehira, T. Yoshihara, H. Ishida, Y. Shiina, S. Oishi and S. Tobita, Phys. Chem. Chem. Phys., 2009, 11, 9850-9860.

52 C. Kerzig and M. Goez, Phys. Chem. Chem. Phys., 2014, 16, 25342-25349.

53 M. B. Davies, J. Austin and D. A. Partridge, Vitamin C: Its Chemistry and Biochemistry, The Royal Society of Chemistry, Cambridge, 1st edn, 1991. 